\title{
Video Article \\ Implantation of Total Artificial Heart in Congenital Heart Disease
}

\author{
Iki Adachi ${ }^{1,2}$, David S. L. Morales ${ }^{3}$ \\ ${ }^{1}$ Congenital Heart Surgery, Texas Children's Hospital \\ ${ }^{2}$ Michael E. DeBakey Department of Surgery, Baylor College of Medicine \\ ${ }^{3}$ Cincinnati Children's Hospital Medical Center, The University of Cincinnati College of Medicine
}

Correspondence to: David S. L. Morales at david.morales@cchmc.org

URL: https://www.jove.com/video/51569

DOI: doi:10.3791/51569

Keywords: Medicine, Issue 89, total artificial heart, transposition of the great arteries, congenital heart disease, aortic insufficiency, ventricular outflow tract obstruction, conduit obstruction, heart failure

Date Published: 7/18/2014

Citation: Adachi, I., Morales, D.S. Implantation of Total Artificial Heart in Congenital Heart Disease. J. Vis. Exp. (89), e51569, doi:10.3791/51569 (2014).

\section{Abstract}

In patients with end-stage heart failure (HF), a total artificial heart (TAH) may be implanted as a bridge to cardiac transplant. However, in congenital heart disease (CHD), the malformed heart presents a challenge to TAH implantation.

In the case presented here, a 17 year-old patient with congenital transposition of the great arteries (CCTGA) experienced progressively worsening HF due to his congenital condition. He was hospitalized multiple times and received an implantable cardioverter defibrillator (ICD). However, his condition soon deteriorated to end-stage HF with multisystem organ failure.

Due to the patient's grave clinical condition and the presence of complex cardiac lesions, the decision was made to proceed with a TAH. The abnormal arrangement of the patient's ventricles and great arteries required modifications to the TAH during implantation.

With the TAH in place, the patient was able to return home and regain strength and physical well-being while awaiting a donor heart. He was successfully bridged to heart transplantation 5 months after receiving the device. This report highlights the TAH is feasible even in patients with structurally abnormal hearts, with technical modification.

\section{Video Link}

The video component of this article can be found at https://www.jove.com/video/51569/

\section{Introduction}

The total artificial heart (TAH) was developed as a bridge to cardiac transplant in patients with heart failure $(\mathrm{HF})^{1-4}$. The device is a mechanical circulatory support system which replaces the ventricles, with the purpose of extending life until a suitable donor heart becomes available. Congenital heart conditions may sometimes lead to $\mathrm{HF}$, with patients requiring circulatory support ${ }^{5,6}$. In individuals with CCTGA, the aorta and pulmonary artery (PA) are transposed and the ventricles are inverted ${ }^{7}$. The condition may eventually cause HF due to an inability of the morphologic right ventricle (RV) to pump against systemic vascular resistance ${ }^{8-10}$.

In the case presented here, the patient underwent multiple childhood surgeries for his CCTGA, including a Rastelli repair and a subsequent transvenous dual chamber pacing system. As a teenager, he developed symptoms of HF due to dysfunction of the systemic RV. His condition was complicated by the presence of severe aortic insufficiency (AI) and obstruction of the conduit placed between the left ventricle (LV) to PA. After multiple hospitalizations, including placement with an implantable cardioverter defibrillator (ICD), he advanced to end-stage HF and went into renal failure and cardiogenic shock, requiring intubation and circulatory support.

Although the TAH is designed for individuals with normally-structured hearts, the device was considered the patient's best option for survival considering the severity of his condition and the presence of complex cardiac lesions. To accommodate his transposed arteries and inverted ventricles, the TAH was modified during implantation, and the surgery was successful. Three months after implantation, he was discharged home. He received a donor heart 5 months after implantation with the TAH.

\section{Case Presentation:}

The patient was a 17-year-old Caucasian male with a history of CCTGA, pulmonary atresia, and ventricular septal defect (VSD) (Figures $1 \mathrm{~A}$ and 1B). He had an ascending aorta to main pulmonary artery shunt and epicardial pacemaker placed in infancy. At age 4 , he underwent classical (Rastelli) repair, consisting of VSD closure and placement of a morphologic LV to PA conduit. A residual VSD was closed with an Amplatzer occluder at age 11. He subsequently received a dual chamber transvenous pacing system. At age 16, he was treated with cardiac resynchronization therapy (CRT). 
At age 17, the patient presented to the emergency room (ER) with dyspnea after several days of noncompliance with medication. He reported having a brief episode of chest pain four days prior to the ER visit. The patient was treated with diuretics overnight and was discharged home with medications re-established. Following his hospitalization, cardiac transplantation was discussed in detail with the patient and his family. A complete transplant evaluation was performed and the patient was subsequently listed.

Nine months later, he was admitted to the hospital after an episode of syncope associated with atrial fibrillation. He underwent direct current (DC) cardioversion. Four days after being discharged, he returned to the ER with complaints of chest pain and hemoptysis. He subsequently returned two days later, reporting increased chest pain. Computerized tomography (CT) revealed a pulmonary embolus in a right lower lobe distribution as well as a separate infiltrate. Anticoagulation therapy was initiated. Two weeks later, an implantable cardioverter defibrillator (ICD) was placed following a period of nonsustained ventricular tachycardia.

The patient was discharged nine days after ICD placement but returned four days later complaining of recurrent episodes of dyspnea and palpitations at night when lying down, as well as chronic nausea and decreased appetite. He was re-admitted at this time. His entering medications were digoxin 125 microgram po daily, hydrochlorothiazide $25 \mathrm{mg}$ po daily, warfarin $5.5 \mathrm{mg}$ po daily, enalapril $10 \mathrm{mg}$ po twice daily, furosemide $40 \mathrm{mg}$ po twice daily, and metoprolol $50 \mathrm{mg}$ po twice daily. He was taking Zofran, Colace, Miralax, and Tylenol on an as needed basis. He had no known drug allergies.

Cardiovascular examination was significant for diffuse point of maximal impulse. On auscultation, there was a regular rhythm with a single S1 and fixed, split S2. There was a grade IV/VI, to/fro, systolic/diastolic murmur at the left sternal border. Vitals were heart rate 94 , blood pressure 98/54. Lungs were significant for decreased breath sounds at the right base. Chest x-ray revealed severe cardiomegaly with severely congested lung fields. There was a small right pleural effusion. Bloodwork was significant for progressive hyponatremia, an increase in BNP, and a mild increase in his BUN. The patient was noted to have severely depressed biventricular systolic function, and at least moderate conduit obstruction.

The patient's HF continued to progress in the following few weeks, with multiple admits to the intensive care unit. After an episode of syncope following arrhythmia, he was admitted and placed on milrinone. He remained milrinone-dependent in the hospital but continued to have progressive HF symptoms. Over a $48 \mathrm{hr}$ period, he went into severe HF with renal failure and required intubation. Within 24 hours of intubation, heart dysfunction progressed, with worsening perfusion. He stopped producing urine, his creatinine began to rise, and he became hypotensive.

\section{Diagnosis, Assessment, and Plan:}

The patient was diagnosed with severe HF and cardiogenic shock secondary to CCTGA, status post classic repair. Secondary diagnoses included morphologic LVOT obstruction and VSD. Despite the challenge presented by his congenitally malformed heart, emergent TAH implantation was considered his best chance for survival given his critical condition and complex cardiac adhesions. The alternative solutions of placing extracorporeal membrane oxygenation (ECMO) through the groin or a temporary ventricular assist device (VAD) through the chest or groin were ruled out due to his severe AI and LVOT obstruction. It was felt that stopping the heart and attempting to place a VAD, while also changing the LV to PA conduit and the aortic valve, would not be a viable option and the patient would likely require a biventricular assist device (BiVAD). Dopamine was added to the milrinone to stabilize the patient's blood pressure prior to the surgery.

The surgical plan included modification of the TAH, such that the right and left pumps were implanted in a parallel orientation instead of the normal criss-cross arrangement (Figures 2A, 2B, and 2C). The major determinants of this unusual orientation were I-looped ventricles (a morphologic left ventricle on the right side and vice-versa) and transposed great arteries, with the aorta anterior and leftward to the pulmonary artery (Figures $\mathbf{3 A}$ and $3 \mathrm{~B}$ ).

\section{Pre-operative Patient Preparation}

1. The patient was given general anesthesia according to standard procedures.

2. He was placed on the operating table in supine position.

3. The right neck line and a right atrial PICC line were removed.

4. He received central access in the left femoral vein and remained with his arterial line.

5. A TEE probe was placed and demonstrated severely depressed biventricular function and significant aortic insufficiency.

\section{Cardiectomy}

1. The patient was positioned, prepped and draped in the normal sterile fashion for the repeat sternotomy.

2. The repeat sternotomy was completed (Figure 4). Immediately noticeable was the extremely enlarged heart and severe adhesions.

3. The diaphragmatic surface was dissected out. It was severely adhered secondary to epicardial wires. The patient would not tolerate significant movement of the heart in trying to reach the inferior vena cava (IVC).

4. The aorta was dissected.

5. The right femoral vein was dissected.

6. Pursestrings were placed in the aorta and femoral vein, heparin was administered, and the aorta and right femoral vein were cannulated. The tip of the venous cannula was advanced to the IVC.

7. After reaching appropriate activated clotting time (ACT), the patient went onto cardiopulmonary bypass. Since his systemic vascular resistance was markedly elevated due to severe heart failure, aggressive afterload reduction was required to achieve adequate perfusion pressure.

8. The patient was placed with an MPA vent to achieve better decompression of the left heart. However, it became obvious with full flow that this would be a difficult hemodynamic circuit to keep stable primarily due to significant aortic insufficiency.

9. The superior vena cava (SVC) was dissected and cannulated, and the patient was placed on bicaval cardiopulmonary bypass (Figure 5). 
10. The IVC was dissected and the aorta was cross-clamped. This allowed complete decompression of the heart.

11. The aorta and the main PA were divided (Figure 6).

12. The rest of the ventricular surface including the diaphragmatic surface, left atrial gutter, and right atrial gutter was dissected out. The MPA, the PA branch and the proximal PA branches were also dissected.

13. The right ventricle was dissected out, leaving approximately 3-4 mm of muscle below the right AV valve (Figure 7A).

14. The left ventricle was dissected out leaving a 3-5 mm cuff of ventricular muscle below the left AV valve (Figure 7B)

\section{TAH Implantation}

1. Using a large Prolene on an $\mathrm{MH}$ needle, the previously prepared felt strip with Gore-Tex was whip-stitched to fortify the muscular rim of the ventricular muscle cuffs.

2. The atrial quick-connect was trimmed to a 3-4 $\mathrm{mm}$ rim. It was inverted and sewn to the muscular cuff both on the left and right sides, to prevent scalloping (Figure 8).

3. The ventricles were tunneled out just left of the midline and outflow grafts were cut to the appropriate size.

4. The Ao and PA cuffs were sewn using running sutures (Figure 9).

5. The RV to PA conduit and calcified areas were excised.

6. The atrial cuffs were tested for leaks, and then the Ao and PA anastomoses were tested

7. A driveline tunnel was created (Figure 10).

8. The left TAH ventricle was connected to the left atrial cuff and then to the aorta (Figure 11).

9. Then the outflow graft of the Ao was connected to the system and the cross clamp was released slowly (Figure 12a).

10. After this, the TAH right ventricle was connected to the atrial cuff of the RA and then to the PA (Figure 12b).

11. In the final position, the TAH ventricles were aligned in a parallel fashion because the PA graft did not cross over top of the aortic graft due to the TGA anatomy (Figure 13).

12. The right ventricle was de-aired by removing the IVC snare, an active root vent was placed in the ascending Ao and rewarming was completed.

13. The lungs were suctioned and ventilated and the TAH was turned on to de-air the active root vent in the ascending Ao.

14. The patient was taken off cardiopulmonary bypass, and the rate of the TAH was increased.

15. After achieving good hemodynamics and giving protamine, the heart was decannulated.

16. Once hemostasis was achieved, four chest tubes were placed: two Blakes in the mediastinum and posterior mediastinum, one large 40 chest tube in the anterior mediastinum, and a right-angle chest tube in the left chest.

17. A neopericardium made of thin Gore-Tex was created and placed around the device. Once completed, the entire mediastinum was irrigated with copious amounts of warm antibiotic saline solution.

18. The sternum was closed with surgical steel wires, while making sure sternal closure did not cause any compression to the TAH. The skin and underlying tissues were closed in layers.

19. After gaining hemostasis in the groin, this area was closed.

20. The AICD pocket was cultured, the AICD removed, and a small drain was placed in the pocket.

21. The patient tolerated this very complex procedure well and was transferred to the CVICU in a stable hemodynamic and respiratory state. The patient was extubated on postoperative day 2. Unlike management for ventricular assist device, postoperative care TAH does not require inotropes. Fluid control and afterload reduction are the main parts of postoperative care. Anticoagulation was commenced at day 2 per the manufacture's protocol.

\section{Representative Results}

The patient tolerated the TAH procedure well and three months later he was discharged home with a portable driver. Regular TAH outpatient protocol was followed during this time, including physical and occupational therapy.

Six weeks after discharge, he was admitted after hypertensive crisis caused his driver to function poorly, resulting in reduced cardiac output. He was admitted, emergently intubated and switched over to the Big Blue driver, which re-established good output from the TAH. His condition improved over the course of a few days. He was being re-established on warfarin while awaiting a new portable driver when a suitable donor heart became available and the patient was taken for transplantation. Plasmapheresis (net zero, using $4.2 \mathrm{~L}$ of FFP) was performed on the donor heart to clear donor-specific antibodies.

During the surgery, the patient was found to have severe adhesions. The aortic graft was adhered to the posterior sternum and dense adhesions surrounded the TAH. There were no signs of infection. The inferior vena cava (IVC) was very difficult to find because the right ventricle of the TAH was positioned anterior to the IVC. Because of the CCTGA, the PAs were dissected to provide full mobility and the great vessels were then connected in the correct orientation. Due to the abnormal orientation of the heart, especially the left atrium, the lateral side of the IVC was left slightly longer. Surgery was complicated by a significant bleeding from the oropharynx, which necessitated ENT evaluation and packing of the area prior to taking the patient off bypass. A left atrial line was placed through the left atrial anastomosis before coming off cardiopulmonary bypass. Once weaned off bypass, the heart function was quite good. An epicardial echocardiogram demonstrated that all valves were working well, as well as the function of both ventricles. Protamine was given. Meticulous care was taken to achieve hemostasis. The patient tolerated the procedure well and was transferred to the cardiovascular ICU in stable hemodynamic and respiratory state. 


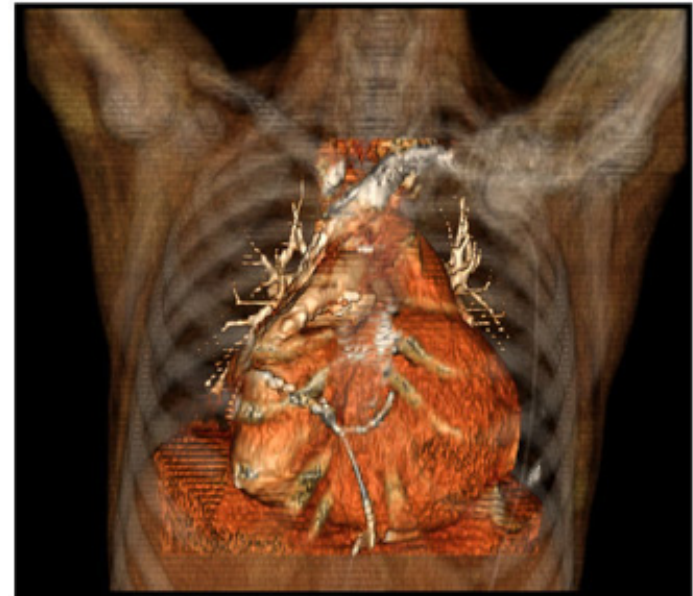

A

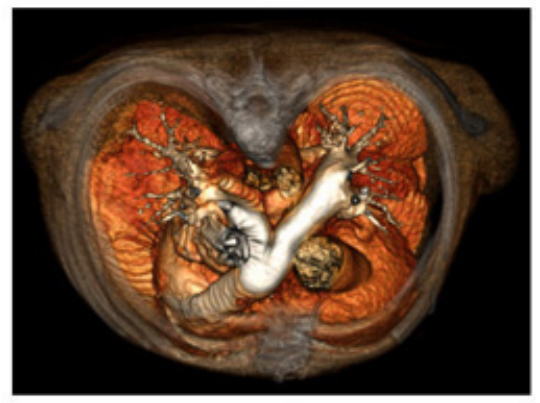

B

Figure 1. Congenitally corrected transposition of the great arteries. Patient CT scans, with coronal view (A) and axial view (B) showing CCTGA with l-looped ventricles (a morphologic left ventricle on the right side and vice-versa) and transposed great arteries, with the aorta anterior and leftward to the pulmonary artery. A conduit was placed between the morphologic left ventricle and the pulmonary artery.

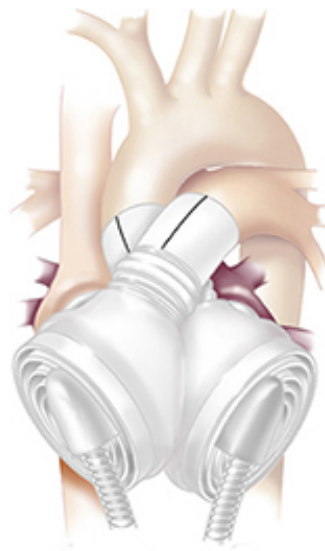

A

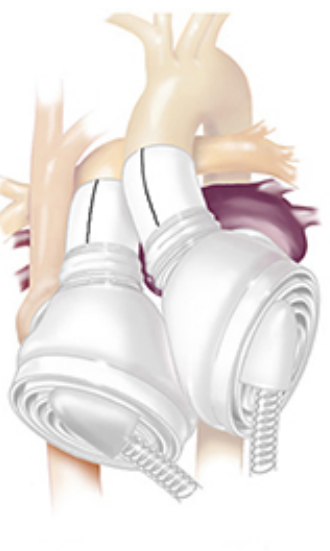

B

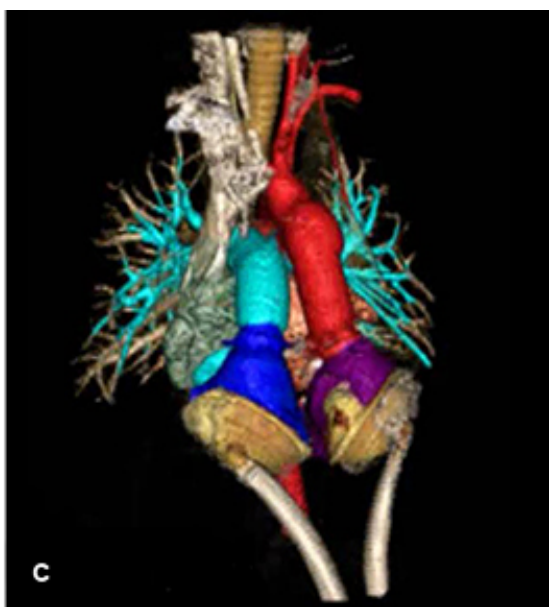

Figure 2. TAH modification. TAH orientation in a normal adult heart, with pumps aligned in a crossed arrangement (A), and modified orientation in a heart with CCTGA (B). Due to abnormal orientation of the great vessels, the pumps need to be in parallel orientation, as seen in the CT (C). 

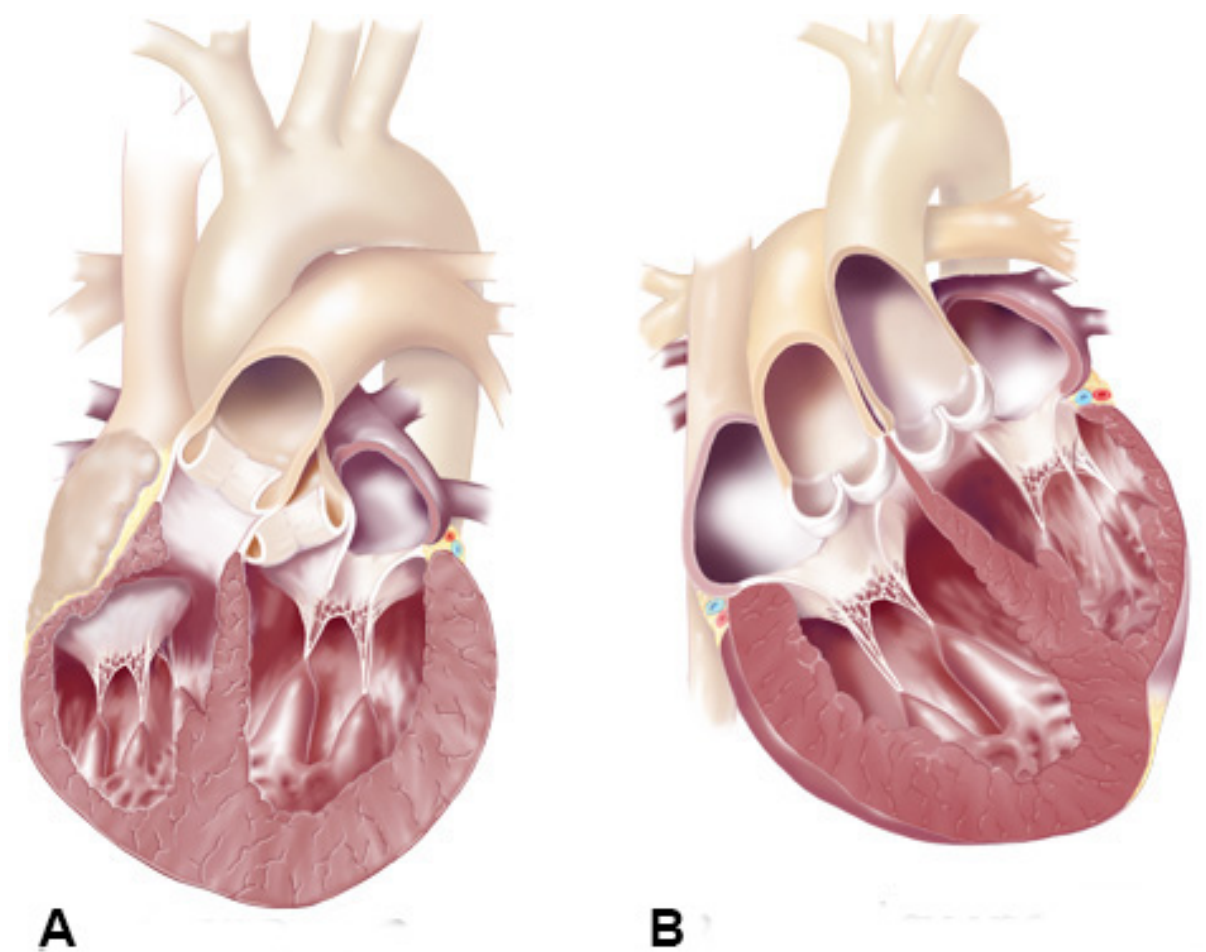

Figure 3. CCTGA Anatomy. Normal arrangement of great vessels and ventricles (A), and the anatomical abnormality in CCTGA (B), with the Ao and PA transposed and the ventricles inverted.

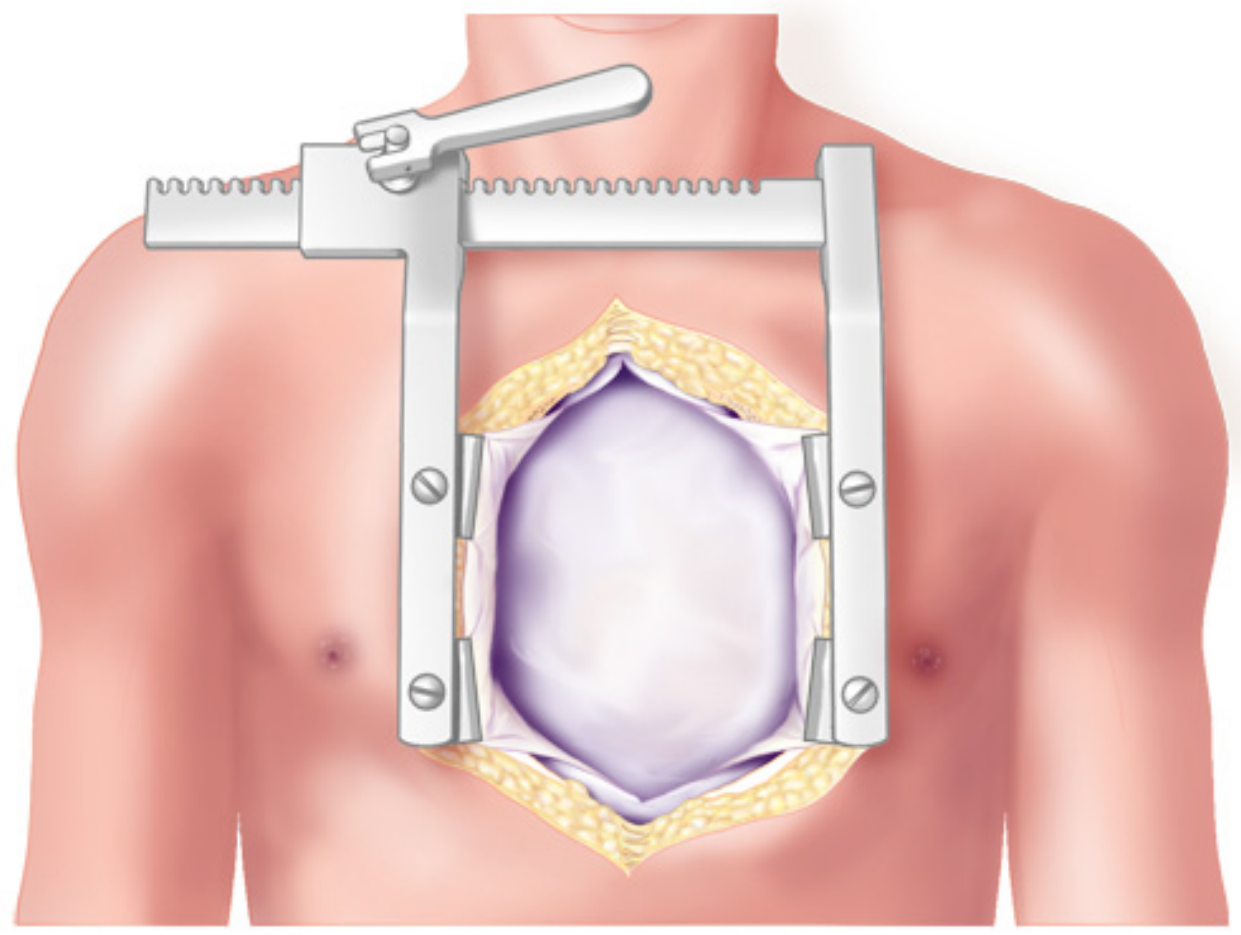

Figure 4. Median Sternotomy. TAH implantation procedure began with a 5th repeat median sternotomy. 


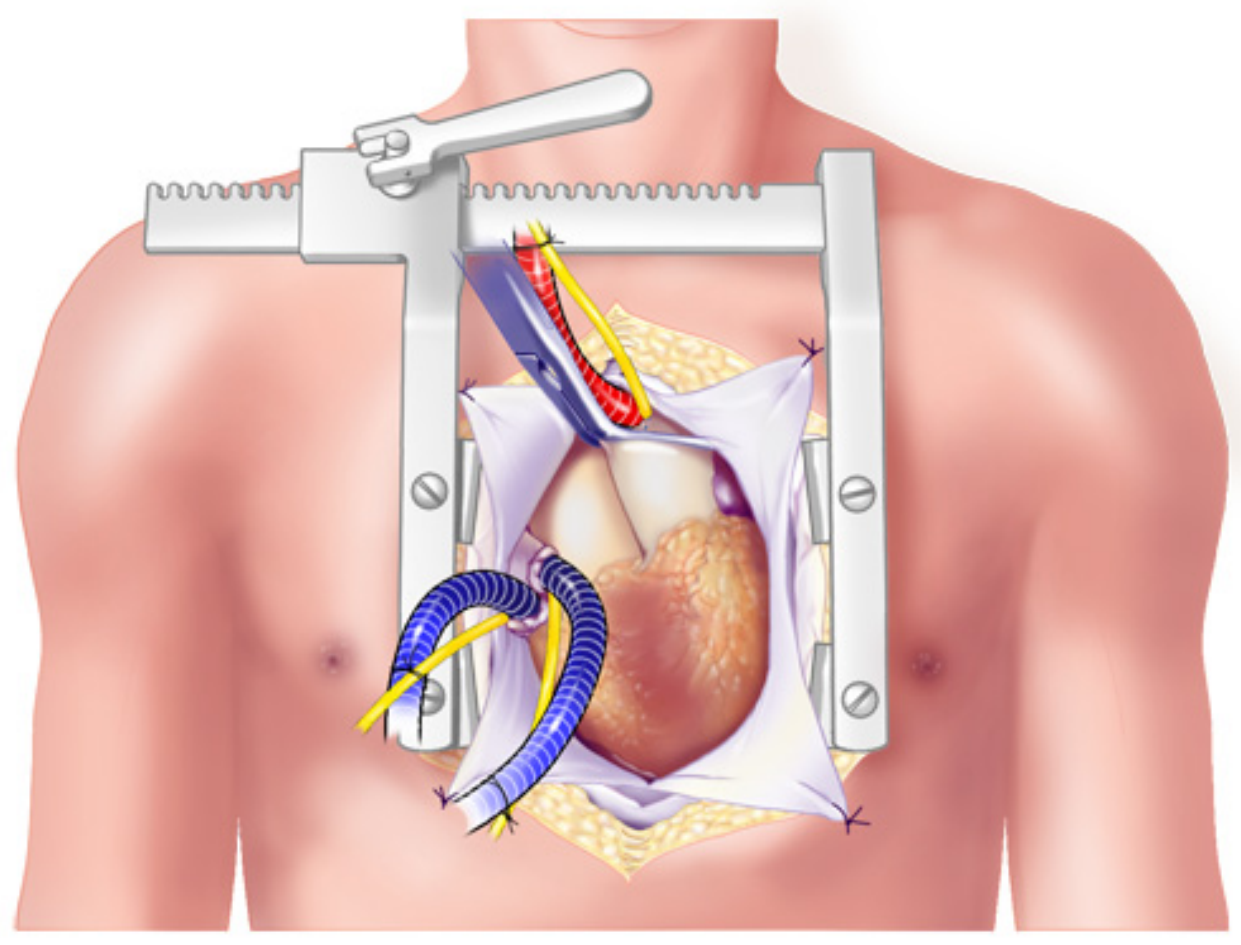

Figure 5. Cannulation. The vessels were cannulated and the patient placed on bicaval cardiopulmonary bypass.

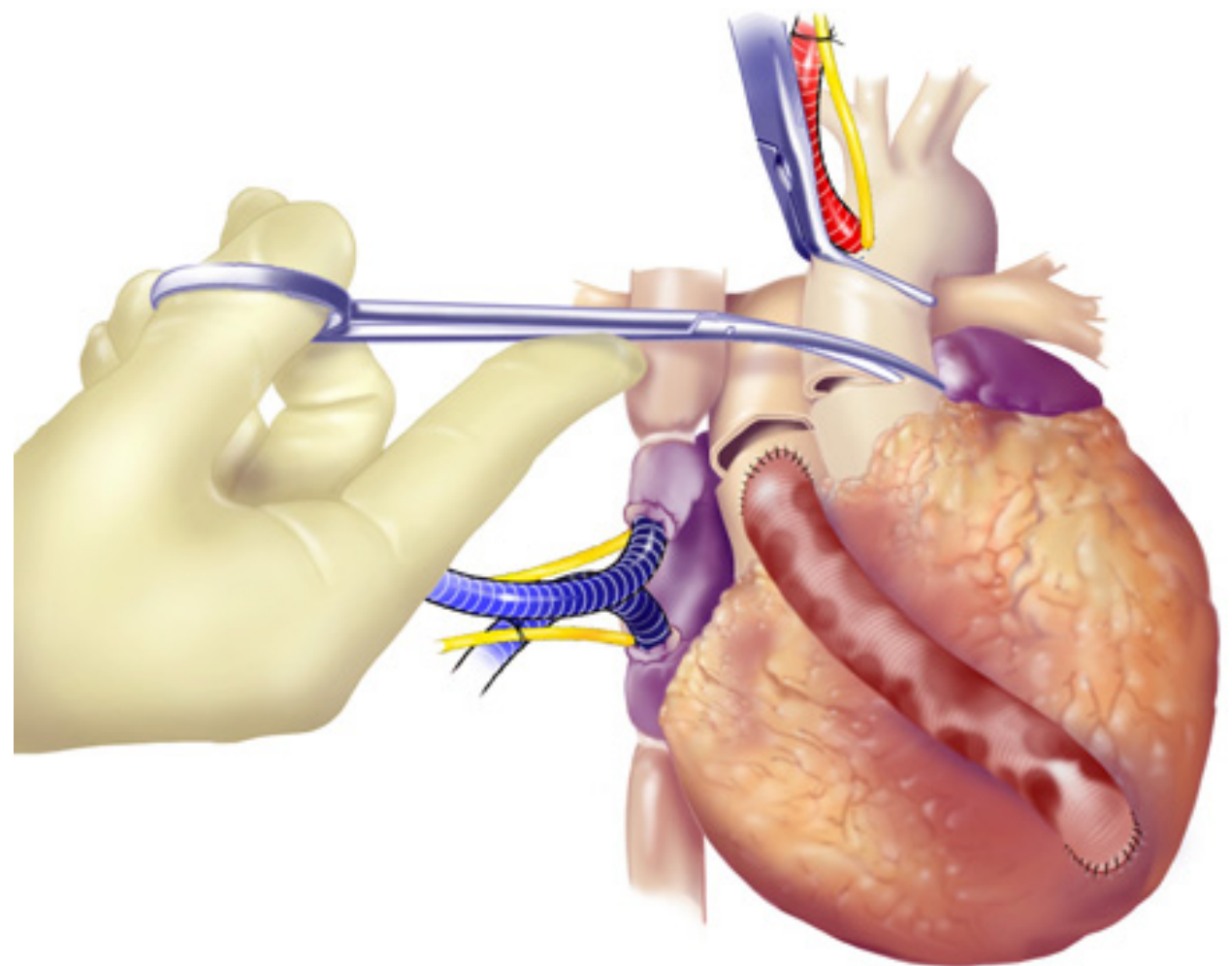

Figure 6. Division of the Aorta and the main pulmonary artery. The aorta was clamped to stop the heart, prior to starting cardiectomy. The aorta and the main pulmonary artery were divided after aortic cross-clamping. 

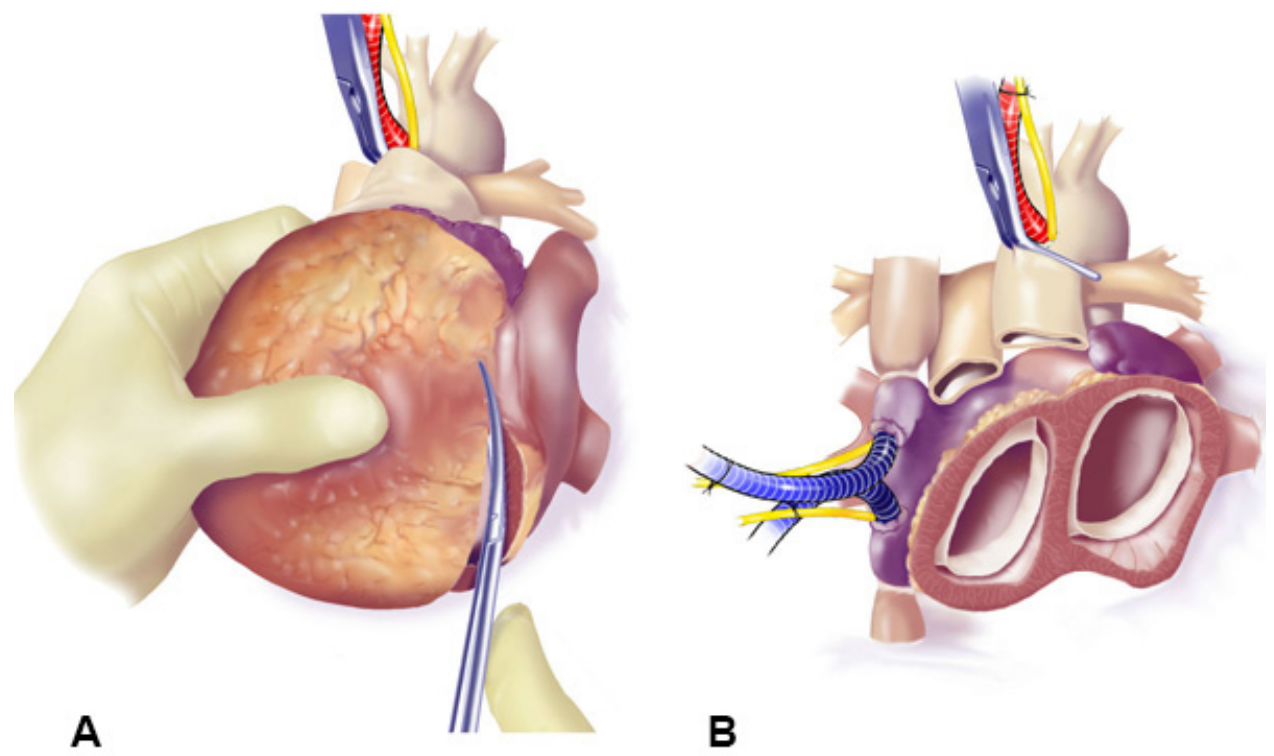

Figure 7. Excision of the Ventricles. The ventricles were excised (A), leaving a 3-5 mm cuff of ventricular muscle below the left and right AV valves (B).

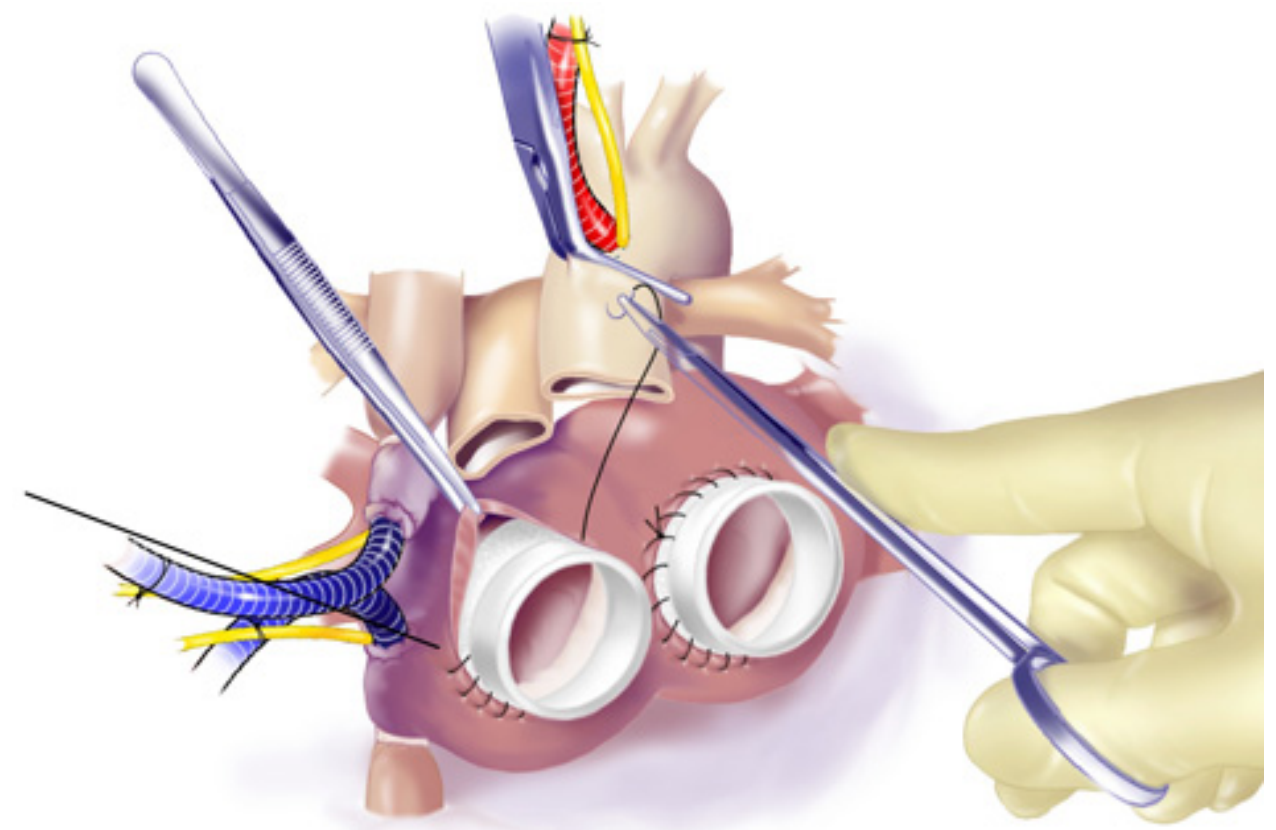

Figure 8. Suturing of Atrial Cuffs. After closing the coronary sinus and left atrial appendage to prevent thrombus formation, the atrial cuffs were trimmed and sutured carefully with running suture. 


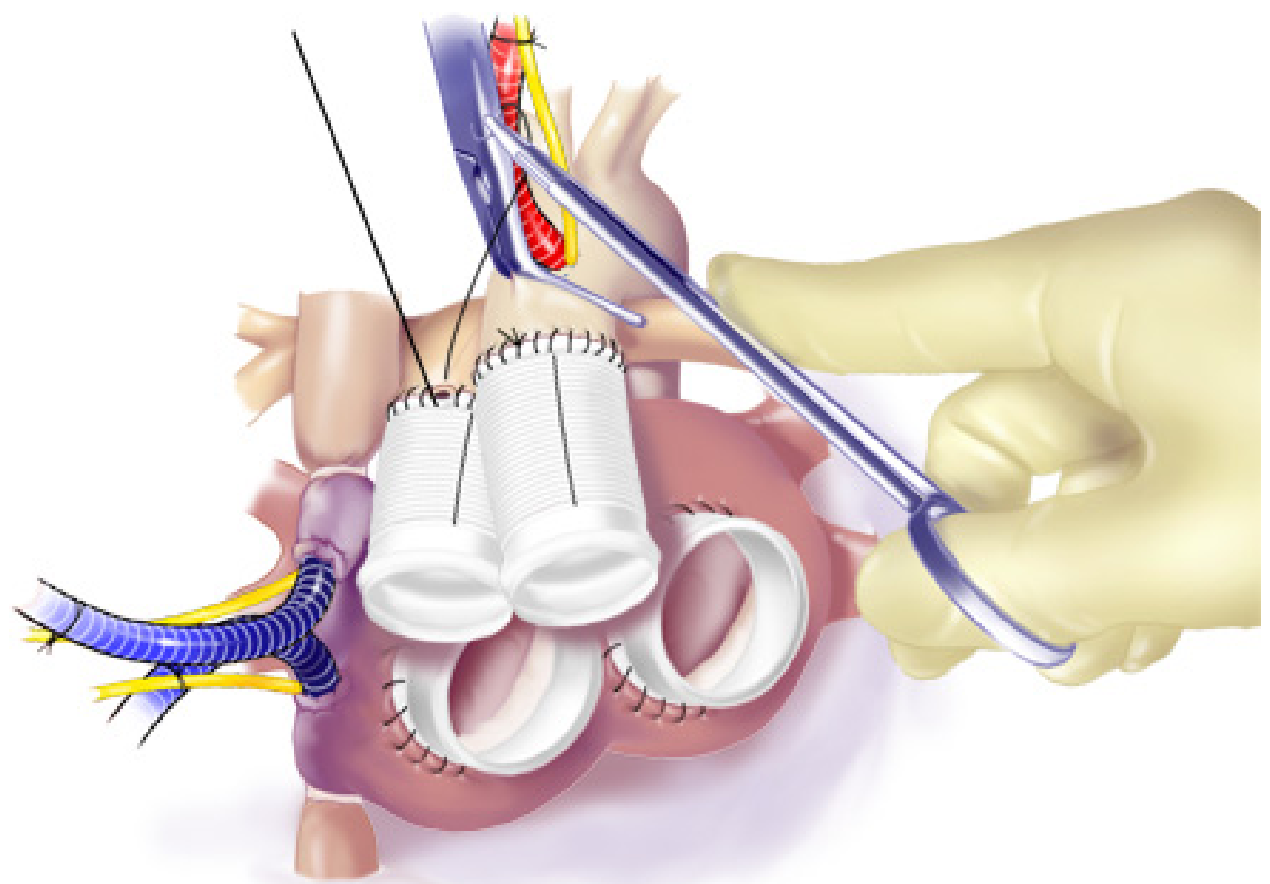

Figure 9. Anastomosis of Outflow Grafts. Running suture was used to anastomose outflow grafts to the Ao and PA.

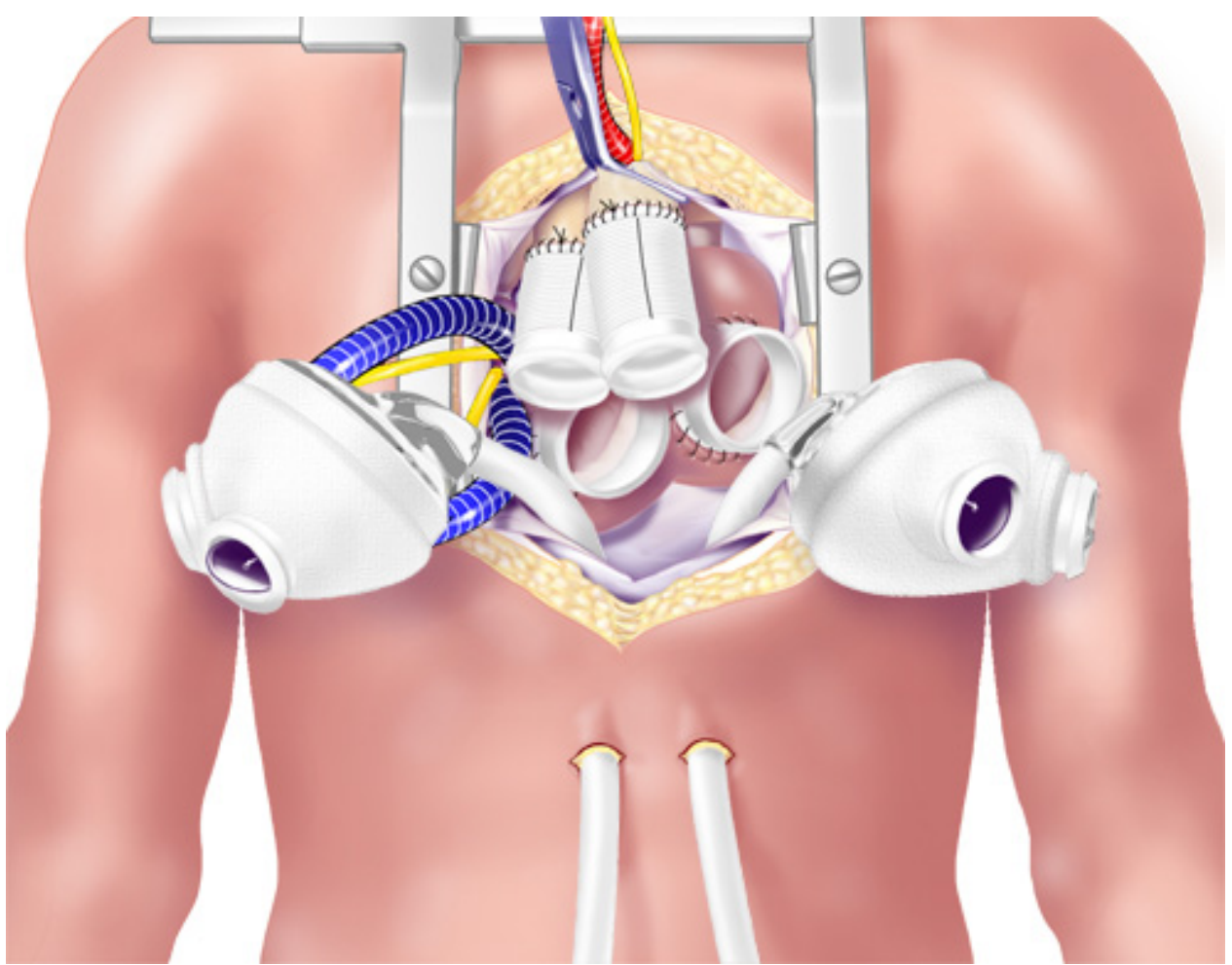

Figure 10. Completed Anastomosed Outflow Grafts. The TAH ventricles were brought into the field. The drivelines were passed through the skin. 


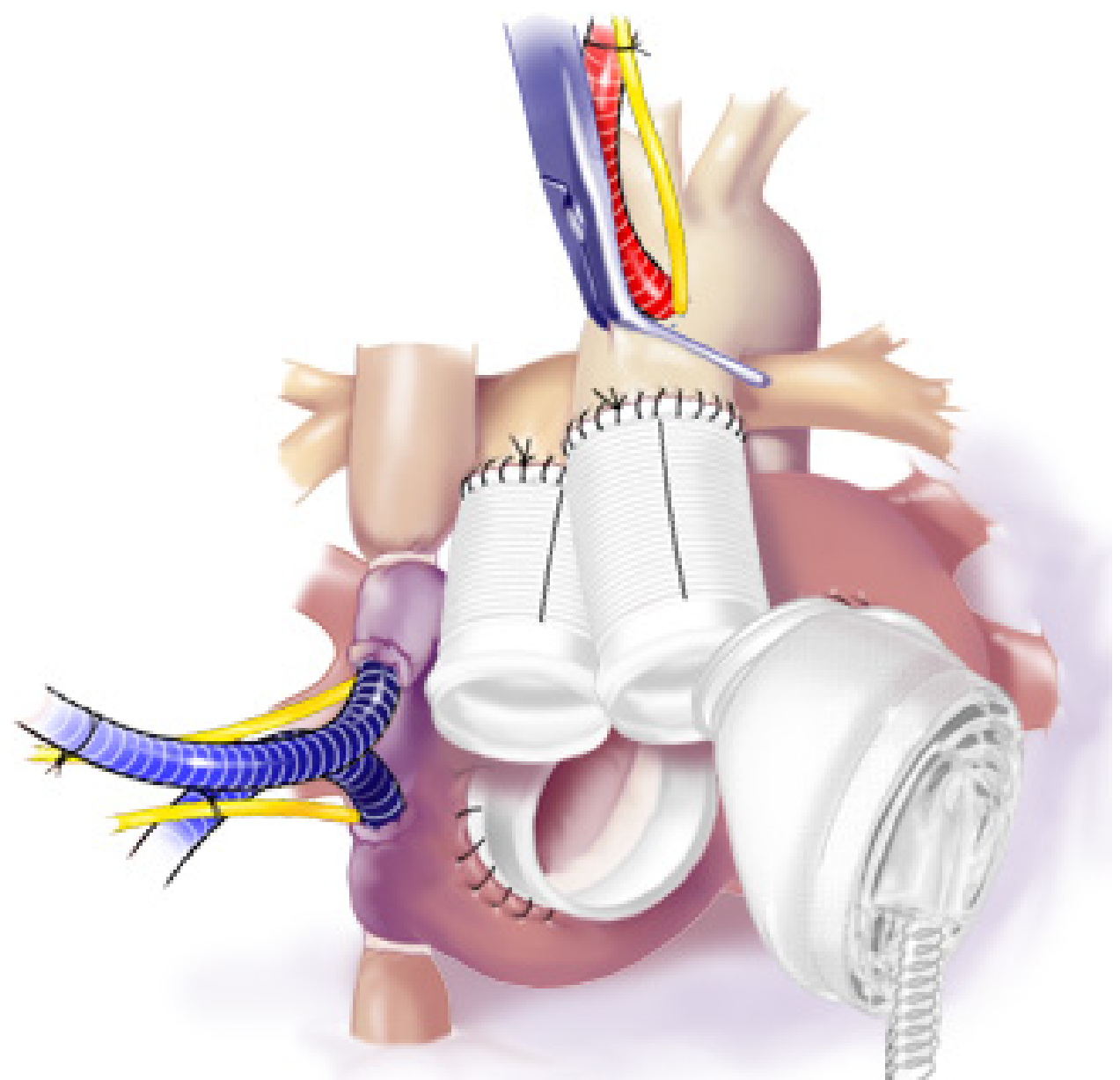

Figure 11. Attachment of Driveline Tunnels. Starting with the left side, the TAH was connected to the atrial cuff and then to the aorta. 


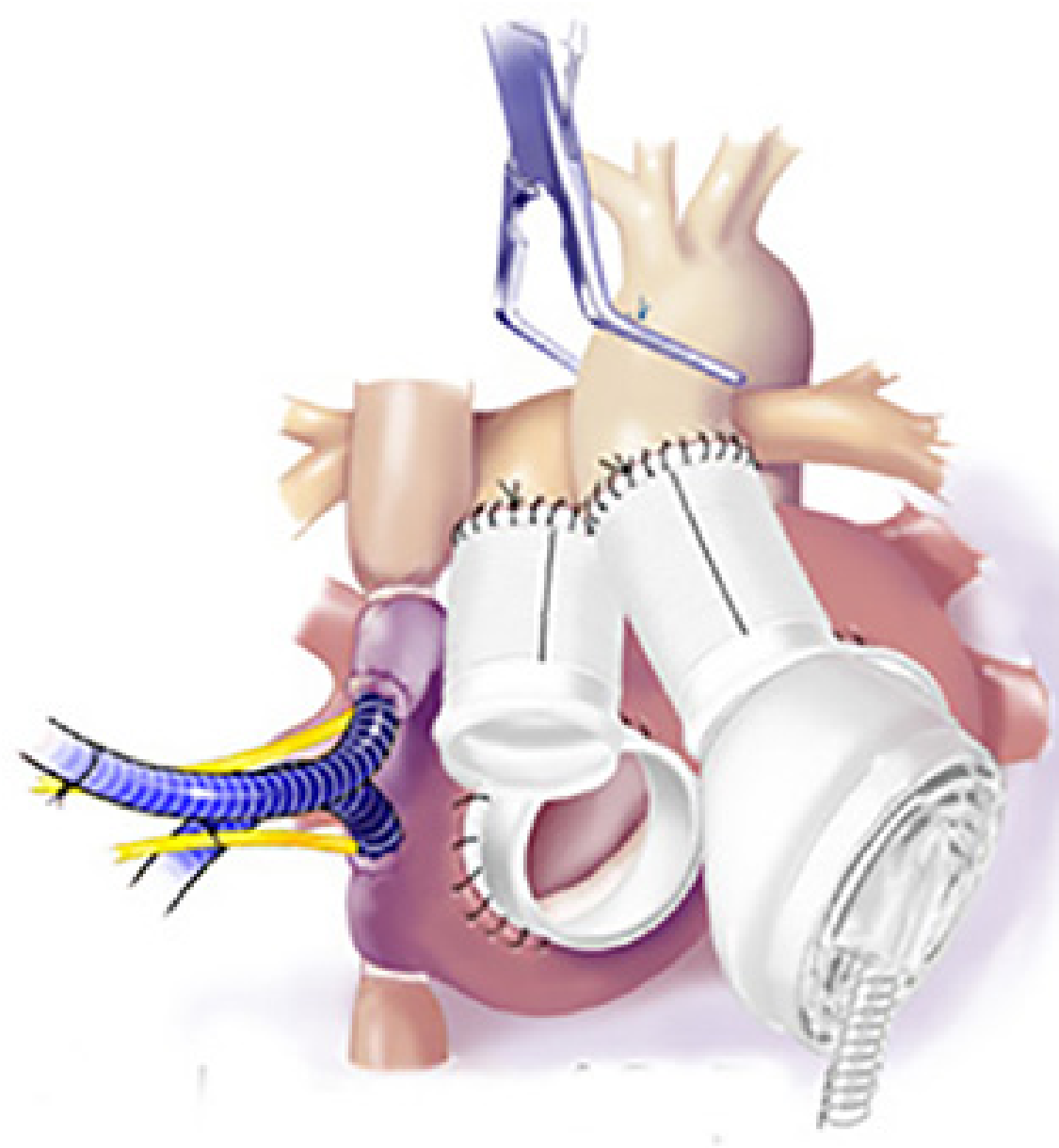

Figure 12. Release of Cross Clamp. After the left TAH ventricle was connected to the left atrial cuff and the aorta, the outflow graft was connected to the system and the cross-clamp was released slowly. 


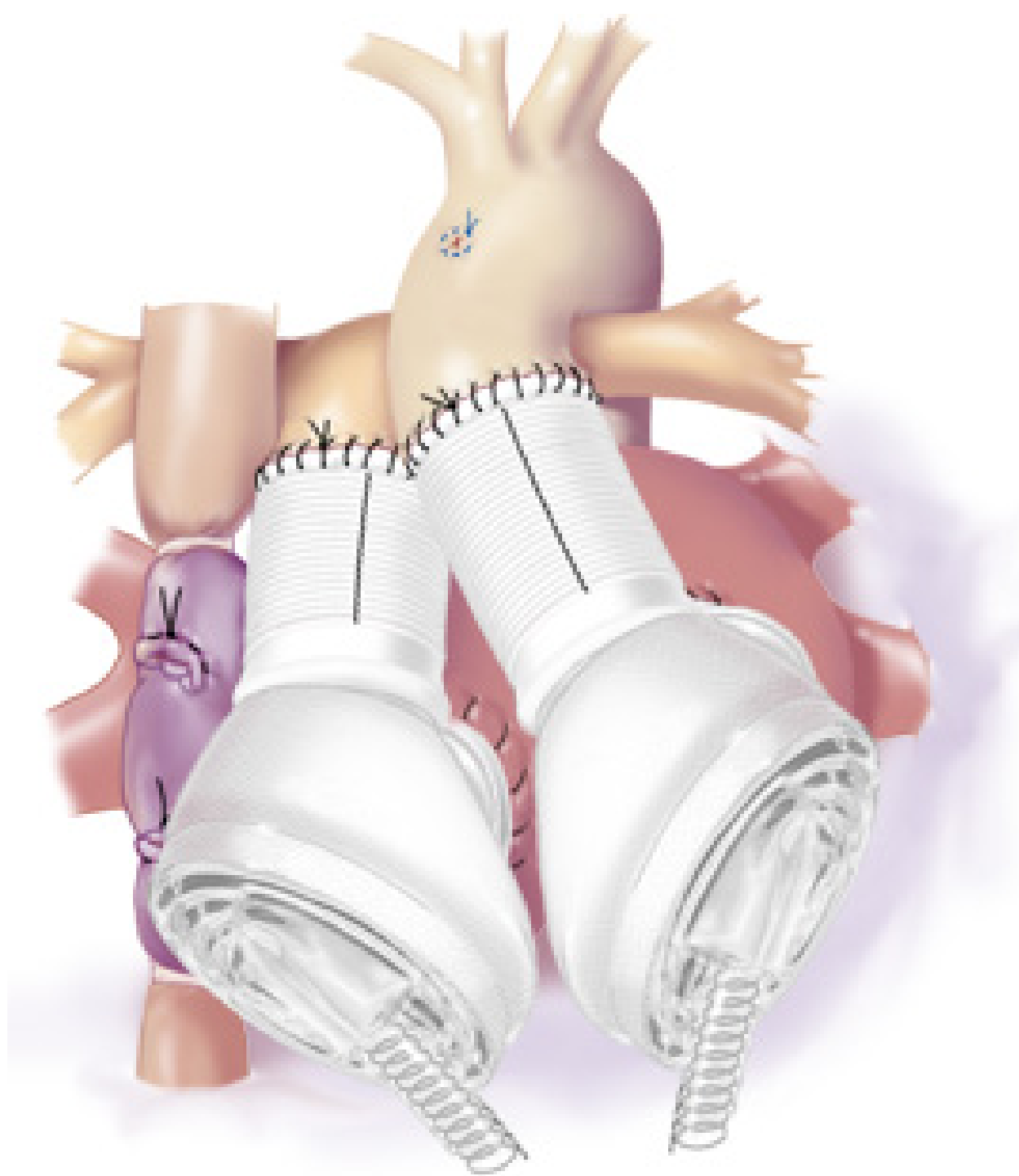

Figure 13. Final TAH position. The TAH pumps were oriented in a parallel fashion to accommodate the TGA anatomy.

\section{Discussion}

The TAH is designed as a bridge to cardiac transplant for patients with normally structured hearts. It enables patients to regain health and stamina as they await a donor heart. Previously, patients with malformed hearts were not considered candidates for receiving a TAH due to the challenges presented by the unusual anatomy. This article highlights that the TAH, with modification, is a viable option for patients with CCTGA a congenital condition which features I-looped ventricles and transposed great arteries in the setting of usual atrial arrangement. Implanting the TAH in a patient with CCTGA requires a technical modification, with the right and left pumps implanted in a parallel orientation instead of the typical crossed arrangement.

The report also demonstrates that the TAH may provide a simpler solution than placement of VADs in critically ill patients with complex cardiac lesions. In this case, the patient had severe Al and obstruction of a conduit between the LV and PA. The TAH was considered a better option than support with a VAD, which would have required multiple concomitant surgical procedures. The patient in this case report was able to return home and regain health and strength prior to being successfully bridged to heart transplantation five months after receiving the TAH.

With advances in medical and surgical treatment, increasing numbers of patients with CHD are surviving to adulthood ${ }^{11,12}$. The prevalence of adults with severe CHD increased $85 \%$ between 1985 and $2000^{12}$. The population of adults with CHD now outnumbers that of children with $\mathrm{CHD}$, and a significant subset of this adult population has an increased risk of $\mathrm{HF}^{13}$. As demonstrated in this case, the TAH provides an additional option for CHD patients with HF. In the light of the increasing number of adult patients with HF due to congenital heart disease, this case should represent the beginning of the new era of mechanical circulatory support for those with malformed hearts. 


\section{Disclosures}

Production of this video-article was sponsored by SynCardia.

\section{References}

1. Kirsch, M., et al. SynCardia temporary total artificial heart as bridge to transplantation: current results at la pitié hospital. Ann Thorac Surg. 95 (5):1640-6. DOI: 10.1016/j.athoracsur.2013.02.036, (2013).

2. Copeland, J., et al. Total artificial hearts: bridge to transplantation. Cardiol Clin. 21 (1), 101-13, (2003).

3. Roussel, J., et al. CardioWest (Jarvik) total artificial heart: a single-center experience with 42 patients. Ann Thorac Surg. 87 (1), 124-9. DOI: 10.1016/j.athoracsur.2008.09.048, (2009).

4. Slepian, M., Alemu, Y., Soares J., Smith, R., Einav, S., Bluestein, D. The Syncardia total artificial heart: in vivo, in vitro, and computational modeling studies. J Biomech. 46 (2), 266-75, DOI: 10.1016/j.jbiomech.2012.11.032, (2013).

5. Koyak, Z., et al. Sudden cardiac death in adult congenital heart disease. Circulation. 126 (16), 1944-54, DOI: 10.1161/ CIRCULATIONAHA.112.104786, (2012).

6. Shaddy, R., et al. Applying heart failure guidelines to adult congenital heart disease patients. Expert Rev Cardiovasc Ther. 6 (2), 165-74, DOI: 10.1586/14779072.6.2.165, (2008).

7. Warnes, C. Transposition of the great arteries. Circulation. 114 2699-2709, (2006).

8. Rutledge, J., Nihill, M., Fraser, C., Smith, O., McMahon, C., Bezold, L. Outcome of 121 patients with congenitally corrected transposition of the great arteries. Pediatr Cardiol. 23, 137-145, (2002).

9. Graham, T., et al. Long-term outcome in congenitally corrected transposition of the great arteries: a multi-institutional study. J Am Coll Cardiol. 36, 255-261, (2000).

10. Voskuil, M., et al. Postsurgical course of patients with congenitally corrected transposition of the great arteries. Am J Cardiol. 83, 558-562, (1999).

11. Hoffman, J., Kaplan, S., Liberthson, R. Prevalence of congenital heart disease. Am Heart J. 147 (3), 425-39, (2004).

12. Marelli, A., Mackie, A., lonescu-Ittu, R., Rahme, E., Pilote, L. Congenital Heart Disease in the General Population: Changing Prevalence and Age Distribution. Circulation. 115, 163-172, DOI: 10.1161/CIRCULATIONAHA.106.627224, (2007).

13. DiNardo, J. Heart failure associated with adult congenital heart disease. Semin Cardiothorac Vasc Anesth. 17, 44-54, DOI: 10.1177/1089253212469841, (2013). 